Meta

Journal des traducteurs

Translators' Journal

\title{
La traduction et la culture du passage
}

\section{Aurélia Klimkiewicz}

Volume 50, numéro 4, décembre 2005

Pour une traductologie proactive - Actes

For a Proactive Translatology - Proceedings

Por una traductología proactiva - Actas

URI : https://id.erudit.org/iderudit/019847ar

DOI : https://doi.org/10.7202/019847ar

Aller au sommaire du numéro

Éditeur(s)

Les Presses de l'Université de Montréal

ISSN

0026-0452 (imprimé)

1492-1421 (numérique)

Découvrir la revue

Citer cet article

Klimkiewicz, A. (2005). La traduction et la culture du passage. Meta, 50(4).

https://doi.org/10.7202/019847ar

\section{Résumé de l'article}

Le but de cet article sous forme d'esquisse est de réfléchir sur les réalités professionnelles du monde de la traduction/localisation au prise avec des échanges de plus en plus denses à l'échelle planétaire. L'explication des divergences qui existent entre la traduction dans le contexte traditionnel et dans le contexte de la mondialisation servira à faire dégager le concept de traducteur en tant que passeur, à formuler quatre tâches du localiseur et à cerner la culture du passage indispensable dans l'articulation des différences et leur mise en contact.
Ce document est protégé par la loi sur le droit d'auteur. L'utilisation des services d’Érudit (y compris la reproduction) est assujettie à sa politique d'utilisation que vous pouvez consulter en ligne.

https://apropos.erudit.org/fr/usagers/politique-dutilisation/ 


\title{
La traduction et la culture du passage
}

\author{
AURELIA KLIMKIEWICZ \\ Université de Montréal, Montréal, Canada \\ aurelia.klimkiewicz@umontreal.ca
}

\begin{abstract}
RÉSUMÉ
Le but de cet article sous forme d'esquisse est de réfléchir sur les réalités professionnelles du monde de la traduction/localisation au prise avec des échanges de plus en plus denses à l'échelle planétaire. L'explication des divergences qui existent entre la traduction dans le contexte traditionnel et dans le contexte de la mondialisation servira à faire dégager le concept de traducteur en tant que passeur, à formuler quatre tâches du localiseur et à cerner la culture du passage indispensable dans l'articulation des différences et leur mise en contact.
\end{abstract}

\begin{abstract}
This paper explores new professionnal realities emerging in translation/localisation world and dealing with growing amount of exchanges on the global scale. In order to define the translator as a border-crosser, to formulate four tasks of the localiser, and finally to delimit culture of bordercrossing, we will explain the differences between traditionnal and new global translation contexts.
\end{abstract}

\section{MOTS-CLÉS/KEYWORDS}

culture, localisation, culture du passage, tâches du localiseur

Si la traduction reste un phénomène complexe et dynamique, c'est parce qu'elle se manifeste différemment selon le modèle culturel auquel elle participe. Dans les sociétés dites ethnocentriques, le texte traduit s'insère discrètement dans la culture réceptrice, tout en sauvegardant une narration collective cohérente et unifiée. Le nivellement ou l'appropriation de l'autre s'accomplit au nom de la supériorité de la culture cible qui fournit une matrice servant à sélectionner, à organiser et à transformer les éléments étrangers. Dans un tel contexte, le traducteur assume le rôle de médiateur qui se plie aux normes de sa propre culture, sans vouloir les questionner ni transgresser. Par contre, là où la traduction est favorable à la rencontre avec l'étranger, elle permet de créer une zone d'échange plus ouverte à la présence de l'autre. Le rôle du traducteur dans ce cas n'est plus le même : situé dans un entre-deux, il devient une figure double, incapable d'appartenir entièrement à l'ordre établi et prêt à introduire les changements qui transforment en permanence son environnement.

Mais ces deux types de traduction, à savoir ethnocentrique et hospitalier, quoique différents en matière de leurs visés, partagent encore quelques éléments semblables : sur l'axe du chronos, ils établissent, tous les deux, un rapport entre un «avant » et un «après », et sur l'axe du topos - une relation entre deux entités culturelles et linguistiques bien distinctes et séparées par une frontière. Autant le traducteur qui endosse le « je » collectif de la société réceptrice que celui qui démultiplie les efforts pour rendre visible la présence des éléments étrangers dans sa version, ils œuvrent dans un lieu qui les oblige à prendre position vis-à-vis du soi ou de l'autre, du propre ou de l'étranger, tout en restant porte-parole de la société d'accueil et travaillant au nom de celle-ci.

Sans aucun doute, la nouvelle réalité socio-économique appelée mondialisation entraîne le monde de la traduction dans une mouvance sans précédent. Non seulement le volume des traductions monte en flèche, mais apparaît une reconfiguration spatio-temporelle à laquelle s'adapte l'activité de traduction : la rapidité de la communication et des déplacements, l'accessibilité quasi instantanée à toutes les cultures du monde, les frontières plus floues, les territoires qui semblent rétrécir et la technologie qui facilitent les échanges à l'échelle planétaire. Comment alors fonctionne la traduction qui, du coup, n'est plus confinée à un échange limité à deux cultures, mais qui, au contraire, s'inscrit dans les déplacements rhizomatiques aux courbes et aux mouvements les plus 
imprévisibles qui forment un réseau semblable à « une carte qui doit être produite, construite, toujours démontable, connectable, renversable, modifiable, à entrées multiples, avec ses lignes de fuites » (Guattari, dans Laplantine et Nouss 2001 : 513-14) ? Quels sont donc les enjeux traductifs liés à cette nouvelle réalité qui propulse le traducteur en dehors de la fixité identitaire et loin d'un découpage territorial réconfortant?

Pour mieux saisir la connexion entre la mondialisation et le monde de la traduction, il serait tout à fait utile d'étudier les implications de cette activité professionnelle dans le monde politique ou économique. Les échanges diplomatiques et commerciaux sont des données concrètes, éloquentes et vérifiables, alors que les contacts culturels non seulement sont moins perceptibles mais suscitent un autre questionnement, puisque d'emblée il s'agit des éléments qui se déposent directement dans l'imaginaire collectif, sans laisser de traces ou de preuves visibles. C'est pourquoi il nous semble important de rapprocher la traduction et la culture, 1'approche d'ordre pragmatique risquant d'évacuer un bon nombre de questions peu lisibles à première vue.

Mais tout d'abord, comment définir la culture, ce phénomène complexe, flou et fuyant ? Selon les marxistes, par exemple, elle est un produit des pratiques sociales. Pour les structuralistes, elle représente un ensemble des structures interdépendantes - linguistique, littéraire, mythologique, entre autres -, offrant des lieux d'échange symbolique pour régulariser l'imaginaire collectif. Quant aux post-structuralistes, ils la définissent en tant que domaine d'idées faisant partie du processus social et servant à expliquer son fonctionnement.

Pour les besoins de notre propos, nous retenons la définition qui ne désigne ni un idéal servant à perfectionner l'individu, ni une documentation présélectionnée, classée et sauvegardée dans des bibliothèques comme une richesse nationale, mais plutôt un phénomène qui désigne une activité, un ensemble des modes de penser, de représenter, d'agir qui dotent l'individu des connaissances pour se comprendre, pour se dire, pour agir. Comme le souligne Michel de Certeau, la culture «consiste non à recevoir, mais à poser l'acte par lequel chacun marque ce que d'autres lui donnent de vivre et de penser » (de Certeau 1993 :123). Dans ce sens, bien que la culture soit un modèle mental du monde, une matrice porteuse de systèmes de croyances et de valeurs, de stratégies cognitives guidant le comportement de l'individu, elle est loin d'être une composante statique de l'univers social. Son aspect régulateur, imposé par le sujet collectif, voile tout simplement son caractère dynamique au nom d'une réalité figée et transparente qui aide à maintenir une cohérence au sein d'une collectivité linguistique et culturelle.

En réalité, comme le précise de Certeau, la culture exige une participation, c'est-à-dire une prise de positions vis-à-vis des significations reconnues socialement : la confirmation, la remise en question ou la transgression. Les produits artistiques, les idéaux ainsi que les valeurs n'ont aucune signification en dehors de l'existence sociale la plus réelle : pour exister et signifier, ils sont actualisés par les membres de la société par le biais, dans le cas des œuvres d'art, de la réception qu'elle soit publication, lecture, re-édition, interprétation, critique, traduction et retraduction. Ainsi, pour comprendre la culture, il faut comprendre tout d'abord comment les valeurs dominantes, les intérêts et les activités prédominants s'actualisent dans les pratiques sociales réelles, autrement, comprendre, par exemple, pour quelle raison nous sélectionnons une œuvre d'art, nous l'utilisons, l'interprétons ou la traduisons d'une certaine manière. C'est là qu'apparaît pleinement l'aspect dynamique de la culture, c'est-à-dire dans la possibilité de questionner, de repenser et éventuellement de transformer les modèles culturels qui se veulent unificateurs, figés, atemporels et au-dessus de la société, excluant du même coup la diversité, le dialogue et le conflit, sans lesquelles la survie d'une culture est impensable.

Comme il a été dit plus tôt, la mondialisation transforme la culture, influe sur la régulation des affaires humaines dans un contexte élargi, sinon planétaire. Aborder la culture par le biais de la pratique traductive offre au moins deux avantages : d'abord, la traduction met en évidence l'ancrage social, historique et idéologique de la culture, et fait voir les interactions qui l'animent autant de l'extérieur que de l'intérieur ; ensuite, elle introduit une distanciation à l'égard des appartenances immédiates au risque de décomposer le réel régularisé par la culture dominante. Du même coup, la traduction nous livre un récit qui dévoile le rapport à soi, à l'autre, c'est-à-dire un mode d'existence basé sur la compréhension du monde et intimement lié à la praxis, à l'expérience directe avec la réalité. Rappelons seulement le rôle que les traducteurs et les interprètes jouaient dans les projets de 
colonisation de même que sous le régime communiste : dans le premier cas, la traduction servait à dénigrer l'indigène, alors que dans l'autre, c'est le passé porteur d'idéologie antagoniste qui était ciblé au nom d'un meilleur monde à bâtir.

Ces allusions historiques illustrent le fait que le traducteur, de par son activité professionnelle, est un agent social et culturel qui pose des gestes concrets ayant un impact sur la réalité, geste qui démontre les connexions entre la culture - ses modèles, ses idées et ses valeurs - et la société qui se charge de les valider, les actualiser à tous les échelons de l'organisation collective. En examinant attentivement une traduction, il est possible de définir si elle participe à l'hégémonie de la culture dominante, si elle négocie la relation entre officiel et subordonné, si elle cache la vérité, si elle dévoilent des stratégies pour abolir ou assouplir les forces du pouvoir, si elle lutte contre l'ordre du monde ou encore si elle ose être un trouble-fête qui transgresse des normes officielles pour déranger ou provoquer.

Mais les défis que doit relever le traducteur pris dans l'engrenage de la mondialisation sont de nature non seulement différente mais plus complexe, ne serait-ce qu'à cause de la confrontation entre le local et le global. Le global signifie l'uniformisation des différences culturelles qui se manifeste à la fois dans le monde matériel (McDonald, popcorn, Internet) et au niveau du récit collectif qui raconte une extraordinaire histoire de l'extension des marchés de consommation à l'échelle planétaire, dont le mot d'ordre est la consommation. Les experts ou les « sachants » (Lyotard 1979 : 19), ceux qui ont les compétences pour produire et raconter ce récit, proposent un monde sans frontières, un monde du progrès, du confort, au centre duquel se trouve un individu vivant dans l'ubiquité, dans la jubilation du présent et dans un univers libéré de toute contrainte et de toute attente. Quant au local, il signifie tout simplement le respect des spécificités de la société cible. La nouvelle tâche du traducteur, ou plutôt du localiseur, serait donc d'apprendre à négocier entre le local et le global, à rendre cohérente l'articulation entre l'idéologie des marchés mondiaux qui privilégie la circulation fluide des capitaux, des biens et, jusqu'à un certain point, des individus, et les sensibilités locales les plus diverses. Du point de vue linguistique, cela obligera le localiseur à favoriser les variantes régionales qui ne correspondent pas nécessairement aux normes des langues majeures standards.

La deuxième tâche du localiseur consistera à accroître les connaissances des cultures d'accueil pour mieux reconnaître le modèle culturel du public-cible et non seulement saisir le sens ou décoder automatiquement les valeurs, les préjugés et les tabous. Au-delà d'un transfert interlinguistique traditionnel, il faudrait plutôt parler d'un transfert trans-culturel qui invite l'agent professionnel à explorer les signes culturels mais aussi à anticiper les présuppositions idéologiques qu'un texte et sa traduction pourraient véhiculer et dont l'impact pourrait se faire sentir à l'échelle régionale ou plus vaste. Il s'agit d'un impératif, car la localisation renverse le mouvement habituel du processus traductif dont le point de départ est le pays étranger (dehors) et le point d'arrivée est la société cible (dedans). Le localiseur, au contraire, perd ses points de repères et peut se trouver dans différentes configurations spatiales : tous les actants du processus, sauf le localiseur, sont à l'étranger, soit dans le même pays soit dans différents pays ; le donneur d'ouvrage vient de l'intérieur alors que le récepteur se trouve à l'extérieur ; le localiseur décide de vivre et de travailler à l'étranger, tout en s'adressant au public de sa culture d'origine ou bien au lectorat de son nouvel environnement, ou encore, aux différents publics assez éparpillés.

Le processus traductif, quant à lui, impose une troisième tâche qui exige une capacité de négocier avec plusieurs actants qui font partie de l'échange professionnel, mais dont les intérêts peuvent être divergents. D'où l'importance de percevoir le traducteur comme un agent actif de l'échange, capable de démultiplier ses interactions avec un grand nombre de participants appartenant aux cultures variées. Visant un public lointain, travaillant pour un client absent/présent, le traducteur sera condamné à la déterritorialisation qui exigera de lui une capacité de s'adapter, de négocier et de s'orienter dans un monde professionnel où la communication n'est plus linéaire, unidirectionnelle ayant lieu dans son contexte d'appartenance, mais décomposée en créneaux de plus en plus denses et complexes.

La quatrième tâche soulève directement les préoccupations d'ordre éthique. Même si la localisation se veut respectueuse de la différence, cette pratique traductive en pleine expansion n'est qu'un mode pervers de traduction ethnocentrique qu'on pourrait appeler idéocentrique. 
L'apparente souplesse de l'idéologie du marché et sa nature consensuelle ne sont, au fond, que des facettes d'une réalité où l'on se sert de la différence pour mieux l'exploiter, en arrachant les individus à leur propre imaginaire, en ébranlant les symbolisations en vigueur qui permettent de se comprendre et de s'orienter dans la vie. Il apparait évident que le contrat social auquel participe le traducteur n'est plus le même. Rappelons que traditionnellement, le traducteur fait correspondre l'original au consensus social, au nom du narcissisme culturel du pays récepteur dont il est le représentant modèle. Mais dans le contexte de la mondialisation, le traducteur n'a plus aucun contrat social à respecter : il s'avère un allié d'un nouvel ordre du monde, au service des plus puissants. Non seulement il risque d'appuyer une idéologie obstinée à conquérir les marchés mondiaux, mais il l'exporte librement ailleurs, étant déconnecté de son espace physique et identitaire immédiat et, surtout, ne partageant aucune alliance avec le récepteur.

Pourtant ces retombées négatives issues de la mondialisation ne devraient pas voiler une autre problématique qui risque d'interpeller les traducteurs d'aujourd'hui : celle de la mouvance, du déplacement, de la communication pluridirectionnelle, de la fragmentation qui caractérisent le chronotope contemporain qui nous impose une certaine expérience du présent et qui invite à une relecture des récits collectifs qui forgent l'identité et la perception souvent fausse et figée des autres.

Professionnellement, le traducteur accepte les règles qui lui sont imposées par le donneur d'ouvrage, et se doit de le servir de son mieux. Mais comme la mondialisation creuse de plus en plus une scission entre le réel et le symbolique, comme elle provoque un dérèglement entre 1'histoire et le récit, - par exemple, dans le cas de l'ex-URSS où l'on assiste à un effritement de grands symboles unificateurs fabriqués par l'État et à une perte de sens qui s'en suit -, le traducteur sera probablement porté à inventer une symbolisation plus souple puisqu'il sera fréquemment obligé de percevoir le monde à travers les yeux de l'autre, à partir d'un autre topos, qu'il ne partage ni avec ses collaborateurs ni avec le public cible. Cette symbolisation contribuera à réconforter la multiplicité, à viser la satisfaction symbolique des segments culturels hétérogènes, refusant en même temps l'homogénéisation et l'impérialisme culturel de l'Occident, et en encourageant l'intégration des entités culturelles périphériques les plus diverses. Cependant, un tel exploit ne peut être réalisé qu'à l'intérieur d'une culture du passage qui assure de maintenir et d'exercer la différence sur tous les plans par le biais des connexions actives entre les éléments disparates pris dans le processus de communication volontaire ou forcé. D'un autre côté, la même préoccupation surgit là où se manifestent des réactions de replie face à la peur de voir son identité diluée à cause d'une démarche globale qui ignore les spécificités locales. Encore une fois, c'est la culture du passage qui permettra de combattre l'esprit tribal, car elle est soucieuse d'établir une sociabilité, une réciprocité, autant sur le plan international (entre les différentes nations) que sur le plan national (à la verticale, entre les ethnies et la population de souche, et à l'horizontal, entre les différentes ethnies). Une telle activité de rapprochement vise à détruire la hiérarchie entre les nations et la stratification des segments des sociétés multiethniques, ainsi qu'à agir sur la représentation de l'identité collective inclusive, multiple et mobile.

Si l'unité chronotopique du monde contemporain s'effrite, si l'appartenance culturelle est loin de fournir un abri identitaire sécuritaire, car l'influence de la culture mondiale bouleverse les modes de penser et de fonctionner habituels, cela signifie que les liens avec le nous collectif se fragilisent au profit des relations plus ponctuelles et plus spécifiques qui auront tendance à se démultiplier. Dans cette situation, la traduction servira à transformer les modèles de communication et à créer des zones d'échange et de passage adaptées aux contextes énonciatifs inédits, imprévisibles ou même peu probables. En d'autres termes, les liens forts et peut-être même solennels qui se tissent d'habitude entre le traducteur et l'auteur ou entre le traducteur et le récepteur, seront confrontés par des formes d'articulation plus souples, plus instantanées, que François Laplantine nomme de tout petits liens qui refusent la grandiloquence, la certitude, l'indéfectibilité au nom « des liaisons plus discrètes, moins appuyées, n'appelant pas un flot de paroles » (Laplantine 2003 : 381). Si le lien explicite et durable qui cimente a besoin d'un médiateur pour le valider, le légitimer et le mettre de l'avant, le tout petit lien discret et fragile qui se noue dans les transitions et les transformations interpelle plutôt le passeur qui ne peut que réinventer à chaque fois des créneaux de communication là où il n'en a pas. Loin de maîtriser le sens de l'original ou le transfert interlinguistique, le passeur tente simplement d'apprivoiser un 
terrain d'entente toujours provisoire et bute constamment contre le sens qui s'échappe dans les zones obscures. L'exercice de traduction consiste dans ce cas à montrer un processus en devenir, un chemin tortueux que le traducteur forge à force de trébucher et de douter de ses capacités, face à des situations inconnues ou imprévisibles.

Comment donc définir la culture du passage ? Est-ce une tierce-culture, c'est-à-dire ni celle de l'Occident ni celle du monde sous-développé, mais une culture émergente qui serait capable de créer de nouvelles alliances, en intégrant les transformations du monde contemporain ? Est-ce donc une culture à inventer qui ne serait pas une culture d'entre-deux comprise comme un simple compromis, une entente consensuelle ? Ou bien est-ce un rapport à l'autre qui se jouerait d'emblée dans la culture, au-delà des enjeux exclusivement politiques, économiques et historiques ? S'il est important pour nous d'insister sur la culture tout en parlant de la traduction dans le contexte de la mondialisation, c'est parce que - comme le souligne Mikhail Bakhtine -, la culture est le seul domaine d'activité humaine qui élimine les rapports de domination, de comparaison, de concurrence. Il ne s'agit pas bien sûr des cultures dites nationales, d'emblée enracinées et hostiles à l'égard des autres, mais d'un espace de création et de médiation qui permet de tisser le récit, non pas celui qui raconte l'aventure de la mondialisation à la poursuite d'un telos, mais un autre, sans origine ni fin, qui met en scène chacun de nous en tant que héros et héroïne, libres de se repenser en fonction des situations et des besoins plus ponctuels qui ne changeront probablement pas le cours de l'Histoire, mais qui risquent à long terme de transformer les rapports humains ainsi qu'entre différents pays et cultures. Au fond, il est si facile d'apprendre à fonctionner dans une société, il est si facile de respecter ses lois, d'être loyal à ses institutions, etc., mais pouvoir participer à la culture, pouvoir se sentir à l'aise dans le récit collectif, pouvoir jouir librement et équitablement de l'univers symbolique qui appartient à l'autre, c'est une autre histoire.

Tant que la culture ne bougera pas, aucune loi ni institution ne pourront combler la distance qui sépare les natifs et les étrangers, les identités homogènes et les identités hétérogènes. Devant ce nouveau défi, la traduction, comme d'habitude, a deux possibilités : soit se cacher derrière le consensus social soit se manifester en creusant les lieux de passage, en réinventant les modèles de communication plus appropriés à la dynamique du monde contemporain.

\section{RÉFÉRENCES}

De Certeau, M. [1980] (1993) : La culture au pluriel, Paris, Seuil.

LAÏDI, Z. [1994] (2000) : Un monde privé de sens, Paris, Fayard.

Laplantine, F. et A. Nouss (2001) : Métissages. De Arcimboldo à Zombi, Paris, Pauvert.

Laplantine, F. (2003) : De tout petit liens, Paris, Mille et une nuits.

Lyotard, F. (1979) : La condition postmoderne, Paris, Les Éditions de Minuit, coll. « Critique ». 